\title{
Análises laboratoriais e garantia da qualidade de medicamentos distribuídos na rede pública de saúde de municípios paulistas: o caso do maleato de enalapril
}

\author{
Laboratory evaluation and quality assurance of drugs distributed \\ in the public health system of São Paulo cities: the enalapril \\ maleate case
}

Jaqueline Kalleian Eserian*

Centro de Medicamentos, Cosméticos e Saneantes, Instituto Adolfo Lutz (IAL), São Paulo, SP, Brasil

\footnotetext{
* E-mail: jaqueline.eserian@ial. sp.gov.br
}

Recebido: 03 out 2019

Aprovado: 28 set 2020

\begin{abstract}
RESUMO
Introdução: 0 monitoramento da qualidade de medicamentos e a investigação laboratorial de queixas técnicas (QT) envolvendo suspeitas de desvios de qualidade são atividades inerentes à vigilância sanitária. Objetivo: Avaliar a qualidade físico-química de comprimidos de maleato de enalapril referentes a um Programa de Monitoramento da Qualidade (PMQ) e provenientes de notificações de QT em municípios de São Paulo. Método: As amostras constituíram-se por seis lotes de comprimidos do PMQ e quatro lotes provenientes de QT, coletadas em 2013 e no período de 2013 a 2016, respectivamente. Foram realizados ensaios de aspecto do produto, determinação de peso, identificação e doseamento do teor de enalapril, uniformidade de doses unitárias e dissolução. Resultados: Para as amostras do PMQ, o teor de enalapril apresentou valores entre $91,3 \%$ e 94,5\%, enquanto o valor de aceitação relativo à uniformidade variou entre 7,7 e 14,6. O valor mínimo de dissolução foi de $85,7 \%$. Em relação às amostras provenientes de QT, o teor de enalapril variou de $91,8 \%$ a $100,3 \%$. Já a uniformidade apresentou valores de aceitação entre 9,5 e 10,0, com valor mínimo de dissolução de 95,3\%. Foi constatada presença de material estranho junto aos comprimidos de uma das amostras. Conclusões: Todas as amostras do PMQ apresentaram resultados satisfatórios, conforme os parâmetros de qualidade preestabelecidos, enquanto uma das amostras provenientes de QT foi considerada insatisfatória devido a uma não conformidade no aspecto. A realização de maior número de PMQ e o incentivo à notificação de suspeitas de QT por profissionais de saúde devem ser promovidos a fim de fortalecer as ações de farmacovigilância no país.
\end{abstract}

PALAVRAS-CHAVE: Vigilância Sanitária; Monitoramento de Medicamentos; Controle de Qualidade; Serviços Laboratoriais de Saúde Pública; Enalapril

\footnotetext{
ABSTRACT

Introduction: Drug quality monitoring and laboratory investigation of product complaints associated to suspicion of quality deviation are essential actions for the health surveillance activity. Objective: To evaluate the physicochemical quality of samples of enalapril maleate tablets from a Drug Quality Monitoring Program and from product complaints in São Paulo cities. Method: The samples were composed by six batches of tablets from the Monitoring Program and four batches from the product complaints, collected in 2013 and from 2013 to 2016, respectively. The aspect of the product, weight determination, identification and potency of the active substance, uniformity of dosage units and dissolution tests were performed. Results: Regarding the Monitoring Program, enalapril potency ranged from $91.3 \%$ to $94.5 \%$, while the uniformity of dosage units varied between 7.7 and 14.6. All dissolution results were above $85.7 \%$. For the complaints samples, enalapril potency ranged between $91.8 \%$ and $100.3 \%$. The uniformity test varied from 9.5 to 10.0 , and the lowest value found for the dissolution test was $95.3 \%$. One of the samples was found to
} 
contain foreign matter accompanying the tablets. Conclusions: All samples from the Monitoring Program fulfilled the requirements according to the established quality standards, while one of the samples from the complaints was considered unsatisfactory due to an aspect nonconformity. A greater number of Monitoring Programs along with an incentive for health professionals to report product problems should be promoted in order to strengthen pharmacovigilance actions in the country.

KEYWORDS: Health Surveillance; Drug Monitoring; Quality Control; Public Health Laboratory Services; Enalapril

\section{INTRODUÇÃOO}

A detecção, avaliação, compreensão e prevenção de qualquer evento adverso relacionado a medicamentos (EAM) são o centro das ações de farmacovigilância. Os EAM incluem reações adversas, erros de medicação, uso abusivo de medicamentos, ineficácia terapêutica e desvios de qualidade em medicamentos, podendo causar danos ao paciente e comprometimento do tratamento farmacoterapêutico ${ }^{1}$.

As atividades de farmacovigilância fazem parte do escopo das Vigilâncias Sanitárias (Visa) de todas as esferas (municipal, estadual e federal), cada qual com suas competências espe-

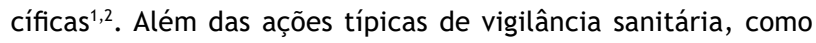
regulamentação e fiscalização ${ }^{1}$, a incorporação de ações de monitoramento da qualidade de medicamentos e notificação e investigação de casos fortalecem a função investigativa inerente ao serviço.

Os Programas de Monitoramento da Qualidade de Medicamentos são constituídos por apreensões planejadas de amostras pela Visa. Os medicamentos são elencados para os programas levando-se em conta a prevalência de seu consumo no mercado, notificações prévias de suspeita de desvio da qualidade e apresentação de resultados insatisfatórios em análises anteriores ${ }^{3,4,5}$.

Comprimidos de maleato de enalapril fazem parte do escopo do Programa Municipal de Verificação da Qualidade de Medicamentos da rede pública municipal de São Paulo, iniciado no ano de 2008 por meio de parceria entre o Laboratório Central de Saúde Pública (Lacen) de São Paulo (Instituto Adolfo Lutz) e a Coordenação de Vigilância em Saúde do município de São Paulo (Covisa). Os medicamentos são coletados pela Covisa nas diversas unidades de saúde municipais e encaminhados ao Lacen para análises fiscais, visando avaliação de sua qualidade físico-química. Atualmente, o programa passa por reformulações que visam fortalecê-lo e torná-lo mais abrangente.

Por outro lado, notificações espontâneas de EAM demandam investigação individual da queixa em questão, constituindo-se uma estratégia primordial para ações de farmacovigilância. As notificações são essenciais para a identificação de reações adversas a medicamentos, erros de medicação, ineficácia terapêutica e desvios de qualidade em medicamentos ${ }^{6}$.

Constitui-se queixa técnica a suspeita de alteração ou irregularidade de um produto por desvio dos parâmetros de qualidade exigidos em seu registro na Agência Nacional de Vigilância Sanitária (Anvisa) ou, ainda, por práticas ilegais, podendo acarretar danos à saúde do paciente ou não ${ }^{7,8}$.
Em período concomitante e também posterior à realização do programa descrito acima, algumas queixas técnicas (QT) relacionadas a comprimidos de maleato de enalapril foram investigadas. As queixas envolviam suspeitas de desvio da qualidade relacionado ao aspecto do produto (presença de material estranho nos comprimidos), alterações físicas (esfarelamento ao retirar os comprimidos do blister) ou ineficácia terapêutica (alteração do teor de substância ativa ou de sua liberação a partir da forma farmacêutica).

O objetivo deste estudo foi avaliar a qualidade físico-química de comprimidos de maleato de enalapril referentes ao programa de monitoramento da qualidade citado e provenientes de notificações de QT em municípios do estado de São Paulo (SP).

\section{MÉTODO}

\section{Amostras}

Foram analisadas na modalidade fiscal seis amostras pertencentes ao Programa de Monitoramento da Qualidade de Medicamentos da Covisa/SP (seis lotes de comprimidos de maleato de enalapril $5 \mathrm{mg}$ e 20 mg distribuídos na rede pública municipal de SP) no ano de 2013.

Quatro amostras provenientes de notificações de QT foram analisadas no período de 2013 a 2016. Uma das amostras foi encaminhada por um serviço de Visa municipal do interior do estado de SP (Q1) alguns meses antes do início das análises referentes ao programa, sendo analisada na modalidade de orientação no ano de 2013 (um lote de comprimidos de maleato de enalapril $20 \mathrm{mg}$ ). As outras três amostras restantes foram encaminhadas pela própria Covisa (Q2, Q3 e Q4) após o início das análises referentes ao programa, sendo analisadas na modalidade fiscal, no período de 2013 a 2016 (três lotes de comprimidos de maleato de enalapril $20 \mathrm{mg}$ ).

A análise fiscal é efetuada em medicamentos com objetivo de comprovar sua conformidade com a fórmula do registro sanitário concedido pela autoridade reguladora do país. As amostras de análises fiscais podem ser coletadas em triplicata ou na forma de amostra única. Já a análise de orientação é realizada quando a natureza, forma de coleta ou finalidade da análise não permitem a realização da análise na modalidade fiscal'.

Todas as amostras eram oriundas do mesmo fabricante (medicamento similar) e foram processadas no laboratório assim que deram entrada no serviço, de acordo com a rotina específica do mesmo. O Quadro 1 contém informações detalhadas relativas às amostras avaliadas. 
Quadro 1. Amostras referentes ao Programa de Monitoramento da Qualidade de Medicamentos da Covisa/SP e provenientes de notificações de queixas técnicas (suspeitas de desvio da qualidade) relacionadas a comprimidos de maleato de enalapril, encaminhadas no período de 2013 a 2016 por serviços de Vigilância Sanitária municipais do estado de São Paulo (Brasil).

\begin{tabular}{|c|c|c|c|c|c|}
\hline Amostra & Dose & Programa de monitoramento & Queixa técnica & Ano & Modalidade de análise \\
\hline P1 & $5 \mathrm{mg}$ & $x$ & - & 2013 & Fiscal \\
\hline P2 & $5 \mathrm{mg}$ & $\mathrm{x}$ & - & 2013 & Fiscal \\
\hline P3 & $5 \mathrm{mg}$ & $x$ & - & 2013 & Fiscal \\
\hline P4 & $20 \mathrm{mg}$ & $\mathrm{x}$ & - & 2013 & Fiscal \\
\hline P5 & $20 \mathrm{mg}$ & $x$ & - & 2013 & Fiscal \\
\hline P6 & $20 \mathrm{mg}$ & $x$ & - & 2013 & Fiscal \\
\hline Q1 & $20 \mathrm{mg}$ & - & Corpo estranho junto aos comprimidos & 2013 & Orientação \\
\hline Q2 & $20 \mathrm{mg}$ & - & Esfarelamento dos comprimidos ao retirar do blister & 2013 & Fiscal \\
\hline Q3 & $20 \mathrm{mg}$ & - & Ineficácia terapêutica & 2014 & Fiscal \\
\hline Q4 & $20 \mathrm{mg}$ & - & Ineficácia terapêutica & 2016 & Fiscal \\
\hline
\end{tabular}

Fonte: Elaborado pela autora, 2019.

\section{Ensaios realizados}

\section{Ensaio de aspecto do produto}

Todos os comprimidos encaminhados (P1 - P6 e Q2 - Q4 = 60 comprimidos; $\mathrm{Q} 1$ = 180 comprimidos) foram inspecionados visualmente quanto à aparência (formato e cor dos comprimidos), ao acondicionamento (embalagem primária, secundária e presença de lacre) e a eventuais não conformidades do produto (presença de material estranho, alterações das características físicas e de cor).

\section{Ensaio de determinação de peso}

Vinte comprimidos foram pesados em balança analítica (Mettler Toledo AL204, Columbus, EUA) para determinação do peso médio. São tolerados até dois comprimidos fora do limite de $\pm 7,5 \%$ em relação ao peso médio. Nenhum comprimido pode estar fora do limite de $\pm 15 \%{ }^{10}$.

\section{Identificação e doseamento do teor de maleato de enalapril}

Utilizou-se cromatógrafo líquido de alta eficiência equipado com detector de ultravioleta (CLAE-UV) (Waters, Milford, EUA), coluna Nucleodur C8 Gravity $250 \times 4,6 \mathrm{~mm}, 5 \mu \mathrm{m}$ (Macherey-Nagel, Düren, Alemanha) ou Zorbax C8 $250 \times 4,6$ mm, $5 \mu \mathrm{m}$ (Agilent, Santa Clara, EUA) e temperatura de $65^{\circ} \mathrm{C}$. A fase móvel foi composta por tampão fosfato de potássio monobásico pH 2,0 (Vetec/Sigma Aldrich, Duque de Caxias, Brasil) e acetonitrila (Vetec/ Sigma Aldrich, Duque de Caxias, Brasil) (60:40). Utilizou-se fluxo de $1,5 \mathrm{~mL} / \mathrm{min}$ e volume de injeção de $50 \mu \mathrm{L}$. A detecção foi realizada no comprimento de onda $215 \mathrm{~nm}$.

As amostras foram preparadas na concentração teórica de 200 ppm em metanol (Carlo Erba, Val de Reuil, França) e tampão fosfato $\mathrm{pH} 2,0$, sendo constituídas de alíquotas (em duplicata) resultantes da trituração dos 20 comprimidos utilizados no ensaio de determinação de peso. A solução de referência foi constituída por padrão primário de maleato de enalapril (Instituto Nacional de Controle de Qualidade em Saúde, Fundação Oswaldo Cruz, Rio de Janeiro, Brasil) preparada com os mesmos diluentes utilizados nas amostras e na mesma concentração.
As respostas das soluções-amostra e de referência foram comparadas por meio do software Empower 3.0 (Waters, Milford, EUA). Os produtos cumprem o teste de identificação se apresentam tempo de retenção do pico de maleato de enalapril do cromatograma das soluções-amostra correspondente àquele da solução de referência. Já o ensaio de doseamento é cumprido se os produtos apresentam de $90 \%$ a $110 \%$ da quantidade declarada de maleato de enalapril ${ }^{11}$.

Ensaio de uniformidade de doses unitárias

Dez comprimidos foram avaliados individualmente quanto ao conteúdo de maleato de enalapril de acordo com o método de quantificação descrito no ensaio de doseamento. 0 ensaio de uniformidade é cumprido se os comprimidos apresentam valor de aceitação $(\mathrm{VA}) \leq 15$. No caso de valores de VA maiores que 15 , mais $20 \mathrm{com}$ primidos são testados aplicando-se novos critérios de aceitação ${ }^{10}$.

\section{Ensaio de dissolução}

Seis comprimidos são avaliados em aparelho dissolutor (Erweka DT $12 \mathrm{R}$, Heusenstamm, Alemanha). Utilizou-se como meio de dissolução $900 \mathrm{~mL}$ de água destilada por cuba $\left(37 \pm 0,5^{\circ} \mathrm{C}\right)$, pás e velocidade de $50 \mathrm{rpm}$. A quantidade de maleato de enalapril dissolvida também é analisada pelo método de quantificação descrito no ensaio de doseamento. 0 critério de aceitação é de no mínimo $85 \%$ da quantidade declarada de maleato de enalapril dissolvida em 30 min $^{11}$.

\section{RESULTADOS}

\section{Programa de Monitoramento da Qualidade}

Todas as amostras apresentaram resultados satisfatórios quanto aos ensaios realizados. 0 aspecto dos comprimidos estava condizente com os critérios adotados. A Figura 1 apresenta o cromatograma obtido no ensaio de identificação e doseamento do teor de maleato de enalapril, no qual é possível observar o mesmo tempo de retenção do pico de maleato de enalapril para a solução amostra e para a solução de referência. Os ensaios de determinação de peso, doseamento do teor de maleato de enalapril, uniformidade de doses unitárias e dissolução apresentaram valores dentro dos limites determinados pelos critérios de aceitação de cada ensaio (Quadro 2). 

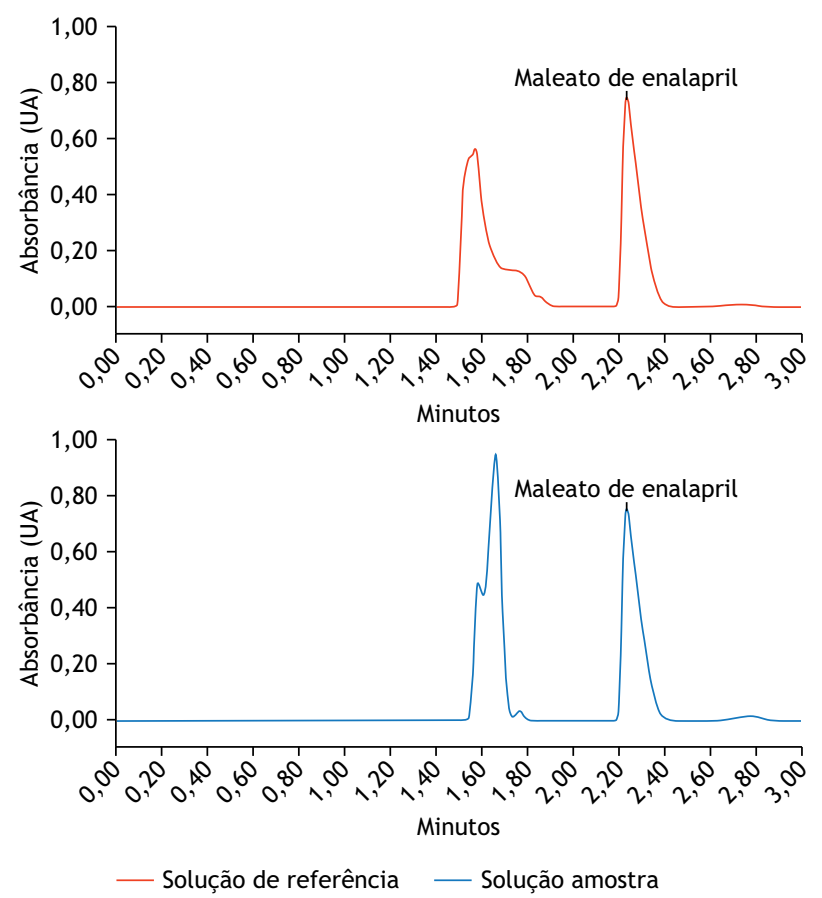

Fonte: Elaborada pela autora, 2019.

Figura 1. Cromatograma obtido no ensaio de identificação e doseamento do teor de maleato de enalapril.
Queixas técnicas (suspeitas de desvio da qualidade)

Entre as amostras provenientes de notificações de QT, uma foi considerada insatisfatória devido à não conformidade no ensaio de aspecto (Q1). Um dos blísteres deste lote apresentou dois alvéolos contendo material estranho sólido solto, de cor acinzentada, localizado entre o blister e os comprimidos. Os resultados referentes às amostras provenientes de notificações de QT são apresentados no Quadro 3. Para a amostra Q1, não foi dada continuidade aos outros ensaios devido à não conformidade constatada no ensaio de aspecto que condenou o lote logo no início da investigação.

\section{DISCUSSÃO}

Este estudo avaliou a qualidade físico-química de comprimidos de maleato de enalapril referentes a um Programa de Monitoramento da Qualidade de Medicamentos municipal e provenientes de notificações de QT, cujos resultados foram satisfatórios para todos os ensaios previstos, exceto pelo ensaio de aspecto na amostra Q1, na qual foi constatada presença de material estranho junto aos comprimidos.

Investigações em farmacovigilância devem ter por objetivo encontrar explicações para o problema e alterar as condições do risco

Quadro 2. Resultados das análises referentes ao Programa de Monitoramento da Qualidade de Medicamentos realizadas nas amostras de comprimidos de maleato de enalapril, encaminhadas no ano de 2013 pela Coordenação de Vigilância em Saúde do município de São Paulo (Covisa/SP), Brasil.

\begin{tabular}{|c|c|c|c|c|c|c|c|c|}
\hline \multirow{2}{*}{ Amostra } & \multirow{2}{*}{$\begin{array}{l}\text { Aspecto do } \\
\text { produto }^{\mathrm{a}}\end{array}$} & \multicolumn{2}{|c|}{ Determinação de peso ${ }^{b}$} & \multirow{2}{*}{$\begin{array}{l}\text { Identificação } \\
\text { de maleato } \\
\text { de enalaprilc }\end{array}$} & \multirow{2}{*}{$\begin{array}{c}\text { Teor de } \\
\text { maleato de } \\
\text { enalapril }(\%)^{\mathrm{d}}\end{array}$} & \multirow{2}{*}{$\begin{array}{c}\text { Uniformidade } \\
\text { de doses } \\
\text { unitárias (VA) }\end{array}$} & \multirow{2}{*}{ Dissolução $(\%)^{f}$} & \multirow{2}{*}{ Resultado } \\
\hline & & Mínima (\%) & Máxima (\%) & & & & & \\
\hline P1 & De acordo & $-2,7$ & $+2,2$ & Positiva & 92,4 & 14,2 & 92,$5 ; 86,8 ; 98,1 ; 94,3 ; 93,8 ; 86,2$ & Satisfatório \\
\hline P2 & De acordo & $-2,6$ & $+1,9$ & Positiva & 91,3 & 12,9 & 102,$2 ; 87,7 ; 91,0 ; 85,7 ; 97,4 ; 87,4$ & Satisfatório \\
\hline P3 & De acordo & $-2,1$ & $+2,1$ & Positiva & 92,6 & 14,0 & 101,$3 ; 94,0 ; 93,0 ; 100,0 ; 101,7 ; 103,4$ & Satisfatório \\
\hline P4 & De acordo & $-7,1$ & $+2,7$ & Positiva & 92,9 & 14,6 & 93,$8 ; 92,5 ; 85,8 ; 96,6 ; 97,9 ; 96,2$ & Satisfatório \\
\hline P5 & De acordo & $-3,3$ & $+3,2$ & Positiva & 94,5 & 7,7 & 103,$1 ; 98,1 ; 98,1 ; 95,1 ; 99,9 ; 93,7$ & Satisfatório \\
\hline P6 & De acordo & $-1,4$ & $+1,9$ & Positiva & 92,5 & 8,3 & 95,$4 ; 97,0 ; 94,4 ; 94,9 ; 95,7 ; 96,1$ & Satisfatório \\
\hline
\end{tabular}

Fonte: Elaborado pela autora, 2019.

Critérios de aceitação: a embalagem lacrada, aparência conforme critérios adotados, ausência de não conformidades; ${ }^{\mathrm{b}}$ até $\pm 7,5 \%{ }^{10}$; ${ }^{\mathrm{c}}$ positiva ${ }^{11}$;

d $90 \%-110 \%{ }^{11}$; e VA $\leq 15^{10} ;{ }^{f} \geq 85 \%$ da quantidade declarada dissolvida em 30 min $^{11}$

Quadro 3. Resultados das análises realizadas nas amostras provenientes de notificações de queixas técnicas (suspeitas de desvio da qualidade) relacionadas a comprimidos de maleato de enalapril, encaminhadas no período de 2013 a 2016 por serviços de Vigilância Sanitária municipais do estado de São Paulo, Brasil.

\begin{tabular}{|c|c|c|c|c|c|c|c|c|}
\hline \multirow{2}{*}{ Amostra } & \multirow{2}{*}{$\begin{array}{l}\text { Aspecto do } \\
\text { produto }^{\mathrm{a}}\end{array}$} & \multicolumn{2}{|c|}{ Determinação de peso ${ }^{b}$} & \multirow{2}{*}{$\begin{array}{l}\text { Identificação } \\
\text { de maleato } \\
\text { de enalaprilc }\end{array}$} & \multirow{2}{*}{$\begin{array}{c}\text { Teor de } \\
\text { maleato de } \\
\text { enalapril (\%) }\end{array}$} & \multirow{2}{*}{$\begin{array}{c}\text { Uniformidade } \\
\text { de doses } \\
\text { unitárias (VA)e }\end{array}$} & \multirow{2}{*}{ Dissolução (\%) ${ }^{f}$} & \multirow{2}{*}{ Resultado } \\
\hline & & Mínima (\%) & Máxima (\%) & & & & & \\
\hline Q1 & $\begin{array}{c}\text { Presença } \\
\text { de material } \\
\text { estranho } \\
\text { em dois } \\
\text { alvéolos }\end{array}$ & - & & - & - & - & - & Insatisfatório \\
\hline Q2 & De acordo & $-1,4$ & $+1,3$ & Positiva & 94,9 & 9,8 & 96,$7 ; 99,4 ; 97,8 ; 103,0 ; 102,4 ; 95,3$ & Satisfatório \\
\hline Q3 & De acordo & $-2,2$ & $+1,8$ & Positiva & 91,8 & 9,5 & 96,$7 ; 96,8 ; 95,4 ; 98,7 ; 96,2 ; 99,1$ & Satisfatório \\
\hline Q4 & De acordo & $-3,7$ & $+3,0$ & Positiva & 100,3 & 10,0 & 105,$3 ; 101,0 ; 100,5 ; 104,8 ; 100,6 ; 101,7$ & Satisfatório \\
\hline
\end{tabular}

Fonte: Elaborado pela autora, 2019.

Critérios de aceitação: ${ }^{\text {a }}$ embalagem lacrada, aparência conforme critérios adotados, ausência de não conformidades; ${ }^{\mathrm{b}}$ até $\pm 7,5 \%{ }^{10}$; ${ }^{\mathrm{c}}$ positiva ${ }^{11}$;

d $90 \%-110 \%{ }^{11} ;{ }^{\text {e }}$ VA $\leq 15^{10} ;{ }^{\mathrm{f}} \geq 85 \%$ da quantidade declarada dissolvida em $30 \mathrm{~min}^{11}$. 
sanitário em questão1. Os EAM constituem-se como um grave problema de saúde pública, impactando na morbimortalidade dos pacientes e elevando os gastos do sistema público de saúde ${ }^{12}$.

Potenciais EAM associados a desvios de qualidade do produto podem ser evitados por meio dos programas de monitoramento da qualidade, nos quais a qualidade do medicamento é monitorada por um período predeterminado de acordo com o plano de amostragem. Constatou-se que os lotes de comprimidos de maleato de enalapril avaliados pelo programa em questão apresentavam qualidade satisfatória para utilização pela população.

As investigações de EAM envolvem as investigações caso-a-caso e as investigações em farmacoepidemiologia de campo. 0 principal fator de desencadeamento das investigações em farmacoepidemiologia de campo, realizada devido a problemas emergenciais em saúde pública associados a medicamentos, é o aumento de casos inesperados de EAM. Desta forma, investigações caso a caso são fundamentais para o processo investigativo inerente às ações de farmacovigilância'.

A investigação caso a caso é realizada quando se recebe uma notificação de EAM por parte das Visa ou pela rede de vigilância sentinela ${ }^{1}$. A observação de desvio dos parâmetros de qualidade preestabelecidos para o produto pode gerar QT de medicamentos. Após realização da notificação, as queixas são avaliadas para classificação do risco sanitário e planejamento da ação, o que desencadeia inspeção, coleta e análise fiscal do produto ou mesmo a adoção de medidas preventivas ${ }^{5}$.

De todas as QT investigadas no presente estudo, somente a amostra Q1 (referente à queixa técnica de aspecto) foi considerada insatisfatória. Queixas que envolvem alteração no aspecto do produto geralmente são constatadas antes mesmo da utilização do medicamento ${ }^{5}$ devido à fácil visualização do desvio por parte do farmacêutico que irá dispensar o produto ou pelo próprio paciente.

Nenhum tipo de desvio da qualidade nas amostras referentes às QT de ineficácia terapêutica foi constatado. Embora a eficácia terapêutica de um medicamento dependa de vários fatores além da qualidade do produto, como uso correto e estado clínico do paciente, é essencial que a investigação de queixas de ineficácia terapêutica inclua a análise laboratorial do produto. Ainda que todos os ensaios associados à análise fiscal sejam importantes, a investigação de casos de ineficácia terapêutica deve focar na identificação e doseamento das substâncias ativas declaradas ${ }^{3,5}$.

O maleato de enalapril é um medicamento comumente prescrito para o tratamento de hipertensão e insuficiência cardíaca, sendo parte do componente básico da Relação Nacional de Medicamentos Essenciais (RENAME) e da Relação Municipal de Medicamentos (REMUME) do município de São Paulo ${ }^{13,14}$. Destaca-se que, quanto mais prevalente o uso de um medicamento, maiores são as chances de QT relacionadas, devido ao maior número de pessoas que o utilizam e consequentemente maior número de unidades do produto em circulação.

A coleta de amostras para análise laboratorial deve ser feita prontamente, uma vez que o resultado das análises pode vir a confirmar os achados farmacoepidemiológicos ${ }^{1}$. Análises realizadas na modalidade fiscal, de acordo com métodos farmacopeicos e com utilização de padrões de referência primários, garantem resultados confiáveis, principalmente quando o laudo da análise indicar um resultado condenatório.

Tanto a farmacovigilância quanto a farmacoepidemiologia têm como objetivo a identificação e a reunião de evidências que corroborem a associação entre uso do medicamento e ocorrência do evento adverso. A observação de evidências consistentes facilita o estabelecimento de inferências de causalidade entre a ocorrência do EAM e a utilização do medicamento em questão, embora a elaboração de tais inferências dependa também de outros fatores ${ }^{15}$.

Resultados satisfatórios em programas de monitoramento da qualidade indicam a segurança e a eficácia do tratamento farmacológico, evidenciando o caráter preventivo de tais ações ${ }^{5,16,17,18,19,20}$. Estudos realizados nos últimos cinco anos mostram que diversos medicamentos que fazem parte do escopo de programas de monitoramento no estado de SP apresentaram qualidade satisfatória no que tange aos ensaios físico-químicos ${ }^{18,19,20}$.

Estudos realizados em municípios de outros estados apontam resultados diversos em relação à qualidade de medicamentos distribuídos pela rede pública. Um estudo realizado no estado da Bahia apresentou resultados satisfatórios para comprimidos de captopril dispensados pela rede pública de saúde de um dos municípios do estado $^{16}$, enquanto outro estudo apontou desvios de qualidade em comprimidos de cloridrato de propranolol e maleato de enalapril distribuídos pela rede pública de saúde de um município de Santa Catarina ${ }^{17}$. A diversidade destes resultados evidencia a necessidade de continuação da realização de estudos nesta área a fim de se manter um espaço para discussão sobre a qualidade e a segurança dos medicamentos distribuídos à população pela rede pública de saúde e consequente qualificação de seus fornecedores.

Já estudos avaliando a qualidade de medicamentos envolvidos em notificações de QT apresentam resultados extremamente variados, uma vez que os eventos decorrentes da suspeita de queixa técnica apresentam características únicas que variam de caso para caso, devendo então receber uma avaliação individual e minuciosa, principalmente em casos que apresentem desfecho clínico grave ${ }^{21,22}$.

Resultados satisfatórios em análises referentes a QT indicam que não há relação causal entre o EAM e a qualidade do produto, embora não se possa afirmar que a utilização do medicamento não esteja relacionada ao EAM. A administração de doses diferentes da prescrita, o não cumprimento do intervalo entre as doses, a interrupção do tratamento, a utilização do medicamento por outra via de administração que não a indicada e o uso off-label do medicamento podem estar relacionados ao EAM, devendo também ser investigados.

Todas as QT recebidas se referiam a comprimidos na dose de 20 mg. Embora as queixas continuassem a ocorrer por três anos após o período de monitoramento estabelecido pelo programa, as investigações apontaram que não havia desvios de qualidade que as 
fundamentassem. A única amostra considerada insatisfatória foi aquela encaminhada por um serviço de Visa do interior do estado de SP previamente à coleta das amostras pertencentes ao programa de monitoramento descrito. Cabe ressaltar que após este ínterim as queixas cessaram, considerando-se a esfera avaliada.

A percepção e a utilização de medicamentos similares, genéricos e de referência foram discutidas em um estudo realizado com 200 pacientes de uma Unidade Básica de Saúde (UBS) de Minas Gerais. Os resultados mostraram que o tipo de medicamento mais conhecido pelos pacientes era o genérico (70\%), seguido pelo de referência (24\%) e similar (1\%). As frequências de utilização dos produtos seguiram a mesma ordem, sendo de $69 \%, 26 \%$ e $3 \%$, respectivamente. 0 estudo ainda mostrou que $67 \%$ dos pacientes não sabiam o que é o princípio ativo de um medicamento, no entanto, atribuíam melhor eficácia ao medicamento de referência (63\%), seguido pelo genérico (26\%), similar $(4 \%)$ e ambos $(7 \%)^{23}$.

Parâmetros cada vez mais criteriosos têm sido estabelecidos para os medicamentos similares nos últimos anos. A Lei $\mathrm{n}^{\circ} 13.235$, de 29 de dezembro de $2015^{24}$, trouxe modificações acerca destes produtos, alterando alguns pontos da Lei $n^{\circ} 6.360$, de 23 de setembro de $1976^{25}$, a fim de equiparar o controle de qualidade dos medicamentos similares ao de medicamentos genéricos. Desta forma, passou a constar na Lei $\mathrm{n}^{\circ} 6.360 / 1976^{25}$ (Art. 21, § $6^{\circ}$ ) que "o medicamento similar [...] deverá ter sua eficácia, segurança e qualidade comprovadas de forma equivalente à adotada para o medicamento genérico".

Independentemente das atualizações regulatórias e da publicação de estudos científicos recentes que apontam resultados satisfatórios para este tipo de produto ${ }^{26,27}$ - em concordância com os resultados gerais observados no presente estudo -, a desconfiança por parte de muitos consumidores e profissionais da saúde quanto à utilização de medicamentos similares parece perdurar.

Pode-se identificar como limitações deste estudo a reduzida quantidade de amostras avaliadas e a falta de maiores detalhes sobre as QT investigadas. Estudos futuros mais abrangentes poderão complementar os achados discutidos nesta pesquisa.

Recomenda-se a firmação de novas parcerias para o estabelecimento de programas de monitoramento semelhantes, com a inclusão de maior número de lotes de maleato de enalapril na dose de $20 \mathrm{mg}$ na oportuna ocasião, uma vez que a seleção dos medicamentos elencados deve considerar, dentre outros fatores, aqueles com maior número de notificações de QT.

Destaca-se que o maleato de enalapril continua a compor o elenco de programas de monitoramento, tal como o Programa Nacional de Verificação da Qualidade de Medicamentos (Proveme), coordenado pela Anvisa em parceria com o Programa das Nações Unidas para o Desenvolvimento (PNUD) e realizado entre os anos de 2016 a 2018.

\section{CONCLUSÕES}

Todas as amostras avaliadas pertencentes ao Programa de Monitoramento de Medicamentos da Covisa/SP apresentaram resultados satisfatórios, conforme os parâmetros de qualidade preestabelecidos, enquanto uma das amostras provenientes de notificações de QT foi considerada insatisfatória devido a uma não conformidade no ensaio de aspecto. 0 fornecimento de evidências resultantes das análises laboratoriais contribui para o estabelecimento de inferências de causalidade, subsidiando a tomada de decisões em vigilância sanitária.

Desta forma, propõem-se o fortalecimento das parcerias entre os serviços de Visa e os Lacens para realização de maior número de Programas de Monitoramento da Qualidade de Medicamentos, levando-se em conta as especificidades, interesses e condições locais. Incentiva-se também a notificação de suspeitas de QT (desvios de qualidade) de medicamentos por parte dos profissionais de saúde, a fim de, em conjunto, fortalecer as ações de farmacovigilância no país.

\section{REFERÊNCIAS}

1. Mota DM. Investigação em farmacoepidemiologia de campo: uma proposta para as ações de farmacovigilância no Brasil. Rev Bras Epidemiol. 2011;14(4):565-79. https://doi.org/10.1590/S1415-790X2011000400004

2. Ministério da Saúde (BR). Portaria de consolidação $N^{\circ} 2$, de 28 de setembro de 2017. Consolidação das normas sobre as políticas nacionais de saúde do sistema único de saúde. Diário Oficial União. 3 out 2017.

3. Silva ACP. O laboratório oficial na avaliação analítica. In: Rozenfeld S, organizador. Fundamentos da vigilância sanitária. 6a ed. Rio de Janeiro: Fundação Oswaldo Cruz; 2000. p. 271-301.

4. Secretaria de Estado de Saúde do Goiás - SES-GO. Monitoramento de medicamentos. Goiânia: Secretaria de Estado de Saúde do Goiás; 2019[acesso 5 set 2019]. Disponível em: http://www.visa.goias.gov.br/post/ ver/128171/monitoramento-de-medicamentos

5. Lombardo M, Eserian JK. A análise da qualidade de medicamentos e o papel do laboratório oficial no contexto da saúde pública. Rev Adm Saude. 2017;17(67):1-14. https://doi.org/10.23973/ras.67.28

6. Varallo FR, Guimarães SOP, Abjaude SAR, Mastroianni $P C$. Causes for the underreporting of adverse drug events by health professionals: a systematic review. Rev Esc Enferm USP. 2014;48(4):739-47. https://doi.org/10.1590/S0080-623420140000400023

7. Agência Nacional de Vigilância Sanitária - Anvisa. Perguntas frequentes: farmacovigilância. Brasília: Agência Nacional de Vigilância Sanitária; 2019[acesso 10 set 2019]. Disponível em: portal. anvisa.gov.br/documents/33868/2895429/ Perguntas+frequentes+\%E2\%80\%93+Farmacovigil\% C3\%A2ncia/f8935efb-7ba4-404e-96a7-271871f5f9d2 
8. Centro de Vigilância Sanitária - CVS. Queixa técnica de medicamentos. São Paulo: Centro de Vigilância Sanitária; 2010[acesso 10 set 2019]. Disponível em: http: //www.cvs. saude.sp.gov.br/apresentacao.asp?te_codigo $=30$

9. Agência Nacional de Vigilância Sanitária - Anvisa. Coleta, acondicionamento, transporte, recepção e destinação de amostras para análises laboratoriais no âmbito do sistema nacional de vigilância sanitária. Brasília: Agência Nacional de Vigilância Sanitária; 2019[acesso 23 jul 2020]. Disponível em: http://portal.anvisa.gov.br/documents/10181/2957432/ Guia+n\%C2\%BA+19-2019+-+vers\%C3\%A30+2. pdf/43c1ad08-004b-4665-881d-6da2177e1f54

10. Agência Nacional de Vigilância Sanitária - Anvisa. Farmacopeia Brasileira. 5a ed. Brasília: Agência Nacional de Vigilância Sanitária; 2010.

11. United States Pharmacopeia Convention - USP. United States pharmacopeia. 35a ed. North Bethesda: US Pharmacopeia Convention; 2012.

12. Sousa LAO, Fonteles MMF, Monteiro MP, Mengue SS, Bertoldi AD, Dal Pizzol TS et al. Prevalência e características dos eventos adversos a medicamentos no Brasil. Cad Saude Publica. 2018;34(4):1-14. https://doi.org/10.1590/0102-311X00040017

13. Ministério da Saúde (BR). Relação nacional de medicamentos essenciais: Rename 2018. Brasília: Ministério da Saúde; 2018.

14. Secretaria Municipal da Saúde de São Paulo. Relação municipal de medicamentos: Remume SP. São Paulo: Secretaria Municipal da Saúde de São Paulo; 2016.

15. Mota DM, Kuchenbecker RS. Causalidade em farmacoepidemiologia e farmacovigilância: uma incursão teórica. Rev Bras Epidemiol. 2017;20(3):475-86. https://doi.org/10.1590/1980-5497201700030010

16. Peixoto MM, Santos Júnior AF, Santos CAA, Caetité Júnior E. Avaliação da qualidade de comprimidos de captopril dispensados em Feira de Santana, BA. Infarma. 2005;16(13-14):69-73.

17. Bianchin MD, Blatt CR, Soares AS, Külkamp-Guerreiro IC. Avaliação da qualidade de comprimidos de propranolol e enalapril distribuídos no sistema público de saúde em uma cidade do sul do Brasil. Cienc Saude Coletiva. 2012;17(2):491-8. https://doi.org/10.1590/S1413-81232012000200022

18. Eserian JK, Lombardo M. Monitoramento da qualidade de comprimidos de clonazepam distribuídos na rede pública estadual de São Paulo e sua contribuição para o sucesso terapêutico. Rev Eletron Farm. 2015;12(4):57-65. https://doi.org/10.5216/ref.v12i4.36761

19. Eserian JK, Lombardo M. Avaliação da qualidade de comprimidos revestidos de diclofenaco sódico dispensados pela rede pública municipal de São Paulo. Infarma. 2016;28(3):185-90. https://doi.org/10.14450/2318-9312.v28.e3.a2016.pp185-190

20. Eserian JK, Lombardo M. Análise da qualidade farmacêutica de medicamentos hipoglicemiantes na eficácia do tratamento de diabetes no âmbito do sistema público de saúde. Cad Bras Med. 2017;30:11-8.

21. Hermanns-Clausen M, Weinmann W, Auwärter V, Ferreirós N, Trittler R, Müller $C$ et al. Drug dosing error with drops: severe clinical course of codeine intoxication in twins. Eur J Pediatr. 2009;168(7):819-24. https://doi.org/10.1007/s00431-008-0842-7

22. Eserian JK, Lombardo M. Investigação de desvios de qualidade em comprimidos de tiamina (vitamina B1) devido à suspeita de ineficácia terapêutica no tratamento de condições neuropsiquiátricas. Vigil Sanit Debate. 2019;7(1):76-80. https://doi.org/10.22239/2317-269x.01249

23. Dias JC, Paula Junior W. Percepções e utilização de medicamentos genéricos, similares e referência por pacientes atendidos na unidade básica de saúde do bairro Major Prates no município de Montes Claros, MG. Rev Farm Fac S Agost. 2015;5(1):29-45.

24. Brasil. Lei $N^{\circ} 13.235$, de 29 de dezembro de 2015. Altera a lei $\mathrm{N}^{\circ} 6.360$, de 23 de setembro de 1976, para equiparar o controle de qualidade de medicamentos similares ao de medicamentos genéricos. Diário Oficial União. 30 dez 2015.

25. Brasil. Lei $N^{\circ} 6.360$, de 23 de setembro de 1976. Dispõe sobre a vigilância sanitária a que ficam sujeitos os medicamentos, as drogas, os insumos farmacêuticos e correlatos, cosméticos, saneantes e outros produtos, e dá outras providências. Diário Oficial União. 24 set 1976.

26. Sousa MN, Silva TP, Teodoro EIS, Lopes-Ortiz MA, Mello JCP, Medeiros-Araújo DC. Estudo de equivalência farmacêutica entre comprimidos de furosemida similares e genéricos. Infarma. 2020;32(1):77-85. https://doi.org/10.14450/2318-9312.v32.e1.a2020.pp77-85

27. Silva TP, Teodoro EIS, Paula MN, Mello JCP, MedeirosAraújo DC. Estudo de equivalência farmacêutica de comprimidos de cloridrato de metformina genéricos e similares. Infarma. 2020;32(2):160-7. https://doi.org/10.14450/2318-9312.v32.e2.a2020.pp160-167

\section{Contribuição da Autora}

Eserian JK - Concepção, planejamento (desenho do estudo), aquisição, análise, interpretação dos dados e redação do trabalho. A autora aprovou a versão final do trabalho. 